FEDSM2005-77367

\title{
A PHASE-FIELD METHOD FOR INTERFACE-TRACKING SIMULATION OF TWO-PHASE FLOWS
}

\author{
Naoki TAKADA Masaki MISAWA \\ National Institute of Advanced Industrial Science and Technology (AIST) \\ 16-1, Onogawa, Tsukuba, Ibaraki 305-8569, Japan \\ Tel: +81-29-861-8232, Fax: +81-29-861-8722, $\quad$ E-mail: naoki-takada@aist.go.jp
}

\author{
Akio TOMIYAMA \\ Graduate School of Science and Technology, Kobe University \\ 1-1, Rokkodai, Nada, Kobe, Hyogo 657-8501, Japan \\ E-mail: tomiyama@mech.kobe-u.ac.jp
}

\begin{abstract}
For interface-tracking simulation of two-phase flows, we propose a new computational method, NS-PFM, combining Navier-Stokes (NS) equations with phase-field model (PFM). Based on the free energy theory, PFM describes an interface as a volumetric zone across which physical properties vary continuously. Surface tension is defined as an excessive free energy per unit area induced by density gradient. Consequently, PFM simplifies the interface-tracking procedure by use of a standard technique. The proposed NS-PFM was applied to several problems of incompressible, isothermal two-phase flow with the same density ratio as that of an air-water system. In this method, the Cahn-Hilliard $(\mathrm{CH})$ equation was used for predicting interface configuration. It was confirmed through numerical simulations that (1) the flux driven by chemical potential gradient in the $\mathrm{CH}$ equation plays an important role in interfacial advection and reconstruction, (2) the NS-PFM gives good predictions for pressure increase inside a bubble caused by the surface tension, (3) coalescence of liquid film and single drop falling through a stagnant gas was well simulated, and (4) collapse of liquid column under gravity was predicted in good agreement with other available data. Then, another version of NS-PFM was proposed and applied to a direct simulation of bubble nucleation of a non-ideal fluid in the vicinity of the critical point, which demonstrated the capability of NS-PFM to capture liquid-vapor interface motion in boiling and condensation.
\end{abstract}

\section{INTRODUCTION}

For last two decades, much attention has been paid to the phase-field model (PFM) as one of the useful tools to well understand complex phenomena involving self organization of mesoscopic structures in multi-component fluids, such as twophase flows (bubbly flow [1], drop breakup [2], RayleighTaylor instability [3], phase change [4,5], etc.), solidification of binary alloys [6] and polymer membrane formation [7]. Based on the so-called Cahn-Hilliard theory [8], PFM describes an interface as a volumetric transition zone with a finite width between pure components (phases), across which all the physical properties vary steeply but continuously. A freeenergy functional, which has a double-well potential of fluid density and depends on square of local density gradient, allows the coexistence of two phases with diffusive interface without imposing topological constraints on interface as phase boundary. In the free-energy theory, surface tension is defined as an excessive energy per unit area induced by the density gradient inside diffusive interface, enabling calculation of the continuous body force without using interfacial curvature and normal vector. As a result, the PFM-based method for twophase flows does not necessarily require conventional algorithms for the advection and reconstruction of interface and continuum surface force model [9-11], unlike front-tracking, level-set and volume-of-fluid (VOF) methods [12-14]. This feature simplifies interface-tracking calculation on a fixed spatial grid. The PFM method therefore has attractive advantages over the other methods, easy implementations of multi-dimensional interface advection and associated heat and mass transfer across the interface [1-5,15].

PFM methods are categorized into two types according to basic equations; a direct numerical method using Navier-Stokes (NS) equations (referred to as NS-PFM hereafter) $[3,4]$, and a lattice Boltzmann method (LBM) [1,2,5] which uses mesoscopic kinetic equations for the velocity distribution of a number density of fictitious fluid particles $[16,17]$. Both types 
have been applied only to two-phase flows with a small density difference because of numerical instability. To overcome the difficulty, Inamuro et al. [1] recently proposed a two-phase LBM which is applicable to incompressible two-phase flows with the density ratio up to 1,000 .

The purposes of this study are to examine PFM for interface-capturing and tracking simulation, and to develop a PFM-based computational method for two-phase flows. First, we propose a NS-PFM by extending the above-mentioned LBM [1] for simulating immiscible, incompressible, isothermal two-phase flows with a high density ratio. Comparing with the LBM, the proposed method would not only save computational cost, but also have flexibility in selection of space and time discretizations. In order to verify the NS-PFM, several interface-tracking simulations of two-phase flows are carried out for fluids with the same density ratio as that of an air-water system. Second, we took heat transfer into account in the NSPFM for direct numerical simulation of phase change of compressible non-ideal fluid in the vicinity of the critical point.

\section{NOMENCLATURE}

$A$ long-range interaction of van-der-Waals fluid particles

$a \quad$ width of liquid column

$B$ short-range interaction of van-der-Waals fluid particles

g gravitational acceleration vector

$g \quad$ magnitude of gravity $(=|\mathbf{g}|)$

$H$ height of liquid column or position of drop in $z$ direction

I second-rank isotropic tensor

$k$ thermal conductivity

$n^{2} \quad$ aspect ratio of liquid column

$\mathbf{P} \quad$ pressure tensor including surface-tension force

$P$, pressure including excess free energy of interface

$T \quad$ parameter of free energy function (and/or temperature)

$t$ time

u flow velocity vector

$x, y, z$ position in Cartesian coordinate system

\section{Greek Letters}

$\Delta t \quad$ time increment

$\Gamma \quad$ mobility of index function $\phi$ in Cahn-Hilliard equation

$\phi \quad$ index function to indicate interface profile

$\eta \quad$ chemical potential

$\kappa_{S} \quad$ surface tension parameter

$\kappa_{\phi} \quad$ interface thickness parameter

$\mu \quad$ viscosity of fluid

$\rho \quad$ mass density of fluid

$\sigma \quad$ surface tension

\section{Subscript}

\section{G gas phase}

$L \quad$ liquid phase

\section{Superscript}

dimensionless time

\section{BASIS OF PHASE-FIELD METHOD (NS-PFM)}

In this section, we briefly state basic equations of the proposed numerical phase-field methods (NS-PFM) for interface-tracking simulations of two-phase flow, in which Navier-Stokes (NS) equations are combined with the phasefield model (PFM).
Isothermal two-phase fluids with a high density ratio

The proposed NS-PFM for immiscible, incompressible, isothermal gas-liquid flows solves a set of a continuity equation, momentum conservation equations, and a CahnHilliard $(\mathrm{CH})$ equation describing time evolution of diffusive interface profile $[1,3,8]$,

$$
\begin{aligned}
& \frac{\partial \rho}{\partial t}+\mathbf{u} \cdot \nabla \rho=0, \\
& \frac{\partial \mathbf{u}}{\partial t}+(\mathbf{u} \cdot \nabla) \mathbf{u}=\frac{1}{\rho}\left[-\nabla \cdot \mathbf{P}+\nabla \cdot\left\{\mu\left(\nabla \mathbf{u}+\nabla \mathbf{u}^{T}\right)\right\}\right], \\
& \frac{\partial \phi}{\partial t}+\nabla \cdot(\phi \mathbf{u})=\nabla \cdot[\Gamma \phi \nabla \eta] .
\end{aligned}
$$

In the $\mathrm{CH}$ equation (3), the scalar variable $\phi$ is an index to describe interface profile [1], which is continuously distributed in the whole flow field. In this study, the chemical potential $\eta$ is derived from a van-der-Waals free energy $[1,5]$ as follows,

$$
\eta=T \ln \left(\frac{\phi}{1-B \phi}\right)-2 A \phi+\frac{T}{1-B \phi}-\kappa_{\phi} \nabla^{2} \phi,
$$

where $\kappa_{\phi}$ is a parameter to control interfacial thickness at $T<$ $8 A /(27 B)$. For simplicity, the mobility $\Gamma$ was set to be constant.

The density $\rho$ is defined as a continuous function of $\phi[1]$,

$$
\rho=\frac{\rho_{L}+\rho_{G}}{2}+\frac{\rho_{L}-\rho_{G}}{2} \sin \left(\frac{\left(\phi_{L}+\phi_{G}\right) / 2-\phi}{\left|\phi_{L}-\phi_{G}\right|} \pi\right),
$$

where $\phi_{G}$ and $\phi_{L}$ are arbitrary thresholds for the index $\phi$ to distinguish the gas and liquid phases. The tensor $\mathbf{P}$ is expressed as,

$$
\mathbf{P}=\left(P^{\prime}-\kappa_{S}|\nabla \rho|^{2}\right) \mathbf{I}+\kappa_{S} \nabla \rho \otimes \nabla \rho,
$$

where $\kappa_{S}$ denotes the strength of surface tension $\sigma$, and $P^{\prime}$ is the effective pressure including the free-energy increase $\kappa_{S}|\nabla \rho|^{2}$. The parameter $\kappa_{S}$ is set to be constant in the whole flow field, and determined from the definition of $\sigma$ on a flat interface [8],

$$
\sigma \equiv \kappa_{S} \int_{-\infty}^{+\infty}|\nabla \rho|^{2} d x
$$

where the direction of $x$ is normal to interface. The viscosity $\mu$ is interpolated between the phases as a function of $\rho[1]$,

$$
\mu=\mu_{G}+\frac{\mu_{L}-\mu_{G}}{\rho_{L}-\rho_{G}}\left(\rho-\rho_{G}\right) .
$$

In this NS-PFM, we adopted conventional numerical techniques mentioned below. The 2or3D space was discretized uniformly with square or cubic cells in a fixed grid, where scalar and vector variables were located in staggered arrangement. The velocity $\mathbf{u}$ and pressure $P^{\prime}$ were calculated using the projection algorithm. The advection term in Eq.(2) was calculated with a $3^{\text {rd }}$ order upwind scheme [18], while that in Eq.(3) was calculated with a forth-order central difference scheme (CDS). Gradients of scalar variables were calculated with a forth-order CDS, while a second-order CDS was applied to the viscous term of Eq.(2). Time marching was based on the second-order Runge-Kutta's scheme with a constant $\Delta t$.

\section{Thermal two-phase flow with phase change}

For simulating thermal two-phase flows, we adopted a full set of NS equations for a non-ideal fluid and the van-der-Waals equation of state [4,5]. They were solved by using the 
MacCormack scheme. Time evolution of total energy $E$ of the fluid is described by the following equations,

$$
\begin{aligned}
& \frac{\partial E}{\partial t}+\nabla \cdot(E \mathbf{u})=\nabla \cdot\left[(-\mathbf{P}+\boldsymbol{\tau}) \cdot \mathbf{u}+k \nabla T-\kappa_{S} \rho(\nabla \cdot \mathbf{u}) \nabla \rho\right] \\
& E=\frac{1}{2} \rho \mathbf{u}^{2}+\rho(c T-A \rho)+\frac{\kappa_{S}}{2}|\nabla \rho|^{2}
\end{aligned}
$$

where $c$ is the specific heat, $\tau$ is the viscous stress in Eq.(2), and the tensor $\mathbf{P}$ in Eq.(6) is obtained by substituting $P^{\prime}$ expressed as follows,

$$
P^{\prime}=\frac{\rho T}{1-B \rho}-A \rho^{2}-\kappa_{S} \rho \nabla^{2} \rho+\frac{\kappa_{S}}{2}|\nabla \rho|^{2} .
$$

\section{NUMECAL RESULTS OF TWO-PHASE FLOW}

This section describes numerical results of two-phase flow obtained with the proposed NS-PFM. All of the simulations have been carried out on a fixed spatial grid with uniform mesh width $\Delta x=\Delta y=\Delta z=1.0$ in Cartesian coordinate system. As for incompressible two-phase flow, density and viscosity ratios $\rho_{L} / \rho_{G}$ and $\mu_{L} / \mu_{G}$ were set at 801.7 and 73.76 respectively, which correspond to those of air-water system at $1 \mathrm{~atm}$. and at room temperature. The other parameters were set as follows; $A=B=1, T=0.293, \kappa_{\phi}=0.1$, and $\phi_{G}, \phi_{L}=$ either 0.2751 or 0.3802 , $|\mathbf{g}|=g=2 \times 10^{-3}, \Gamma=12, \rho_{L}=1, \rho_{G}=1.247 \times 10^{-3}, \Delta t=0.0125$.
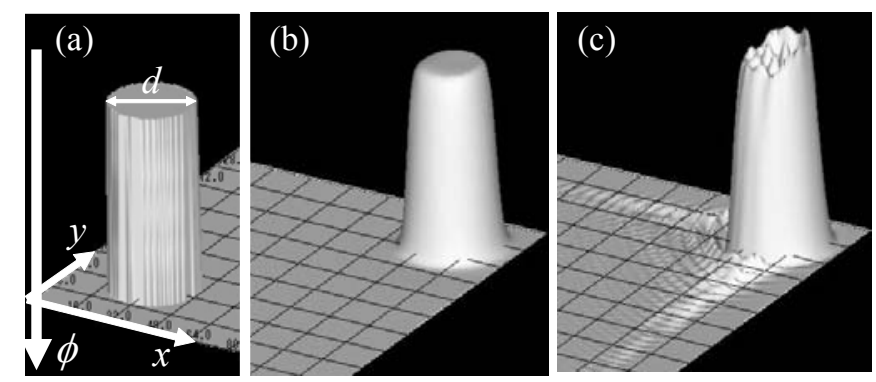

Fig.1 Two-dimensional linear transfer of circular-shaped two-phase interface. (a) Initial condition, and the profiles after transfer for (b) $\Gamma=1$ and (c) $\Gamma=0$.

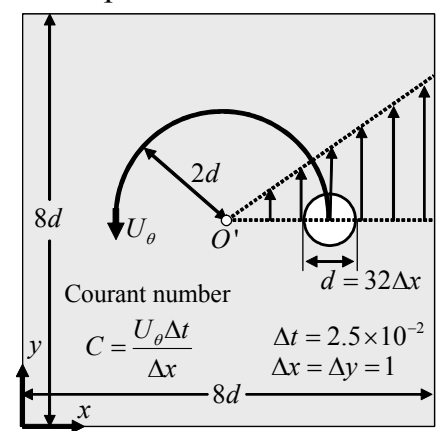

(a) Schematics of domain.

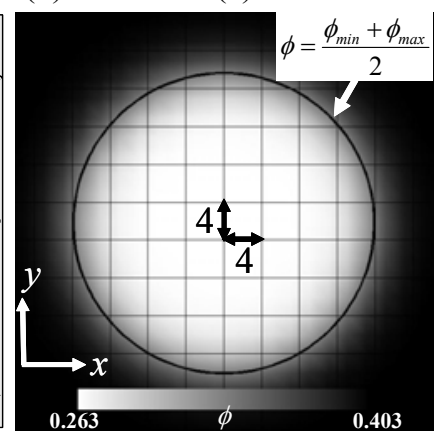

(b) $C=0.1$
Fig.2 Interface profile drawn as a contour line of the index function $\phi$ at $t^{*}=U_{\theta} t / d=12.5$ in $2 \mathrm{D}$ rotation simulation.

\section{Benchmark test on advection of interface}

Two benchmark problems on advection of interface were solved in two dimensions. First benchmark shown in Fig.1 was linear transfer of single circular-shaped interface in a periodic uniform flow with Courant number $C=u \Delta t / \Delta x=4 \times 10^{-3}$, obtained with only Eq.(3) at $u=v=0.1, \Delta t=0.04, \kappa_{\phi}=0.04, \Gamma=0$ and 1. When $\Gamma=1$, the interface profile with an initial diameter $d=32 \Delta x$ has been retained adequately without oscillation until time $t^{*}=u t / d=6.25$. Second benchmark was rotation transfer at $C=0.025,0.05$ and $0.1, \Delta t=0.025, \kappa_{\phi}=0.1$, and $\Gamma=6$ (Fig. 2 ). The fluid volume was conserved within $\pm 1 \%$ error till $t^{*}=20$ (Fig.3).

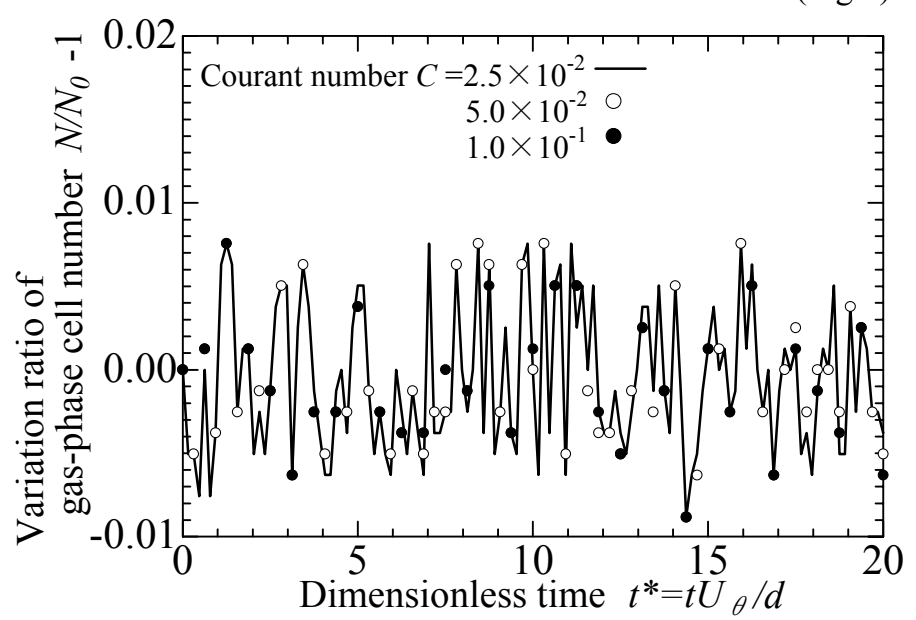

Fig.3 Volume conservation in 2D rotation by a circular vortex.

\section{Surface tension force on gas-liquid interface}

The surface tension force of the NS-PFM was examined in simulation of single bubble neutrally-buoyant in a stagnant liquid. As shown in Fig.4, as the pressure built up uniformly inside the bubble, spurious flow was induced inside interface zone by discretization error of local density gradient in Eq.(6), which is inevitable in the phase-field approach $[1,15,16]$. However, its magnitude was found to be negligible in the following simulations. The excessive pressure was proportional to interfacial curvature, which agreed well with theoretical

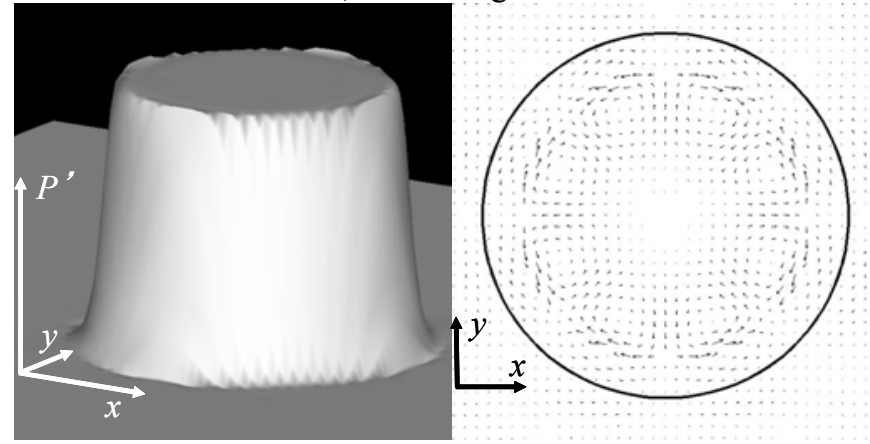

(a) Pressure field

(b) Velocity field

Fig.4 2D neutrally-buoyant bubble in stagnant liquid.

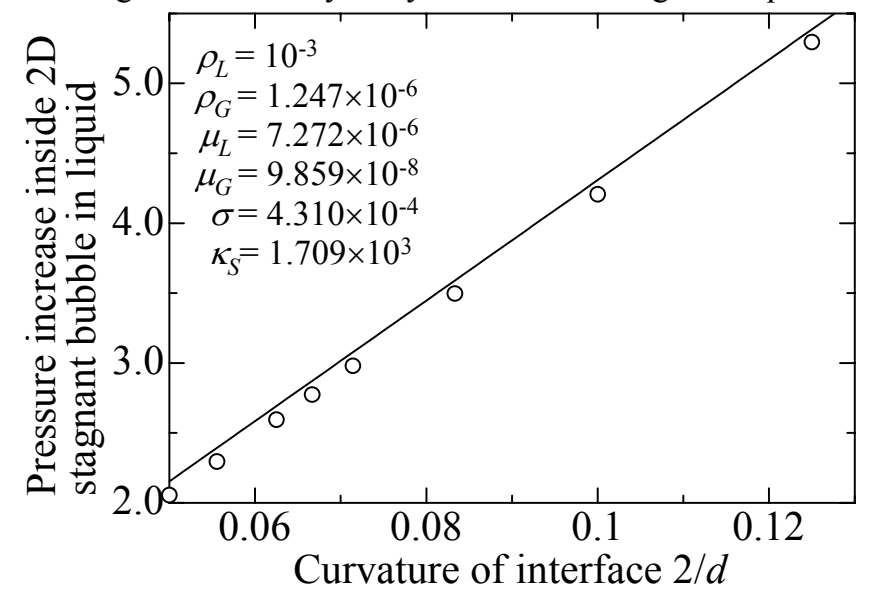

Fig.5 Pressure increase inside bubble neutrally- buoyant in stagnant liquid at $\rho_{L} / \rho_{G}=801.7$ and $\sigma=4.31 \times 10^{-4}$. 
prediction by the Laplace's law (solid line drawn in Fig.5).

\section{Collapse of liquid column under gravity}

To examine the accuracy of the interface-tracking capability, collapse of liquid column in a gas under gravity was calculated by the NS-PFM and compared with available experimental and numerical data $[14,19,20]$. The simulation has been conducted at an aspect ratio of column $n^{2}=H / a=2$ in a

Table1 Parameters in simulation of collapse of liquid column.

\begin{tabular}{|c|c|c|c|c|c|c|}
\hline \multirow[b]{2}{*}{ Case\# } & \multirow{2}{*}{$\begin{array}{l}\text { Aspect } \\
\text { ratio } \\
n^{2}=H / a \\
\end{array}$} & \multicolumn{2}{|c|}{ Width of column $a$} & \multirow{2}{*}{$\begin{array}{c}\text { Surface } \\
\text { tension } \sigma\end{array}$} & \multicolumn{2}{|c|}{ Viscosities } \\
\hline & & $\begin{array}{c}\text { PFM } \\
(\Delta x=1) \\
\end{array}$ & $\begin{array}{c}\begin{array}{c}\text { air\&water } \\
(\mathrm{m})\end{array} \\
\end{array}$ & & $\mu_{G}$ (gas) & $\begin{array}{c}\mu_{L} \\
\text { (liquid) }\end{array}$ \\
\hline 1 & \multirow[t]{2}{*}{2} & $10 \Delta x$ & \multirow{2}{*}{$1.46 \times 10^{-1}$} & $7.11 \times 10^{-5}$ & $1.43 \times 10^{-7}$ & $1.06 \times 10^{-5}$ \\
\hline 2 & & $18 \Delta x$ & & $2.30 \times 10^{-4}$ & $3.47 \times 10^{-7}$ & $2.56 \times 10^{-5}$ \\
\hline
\end{tabular}

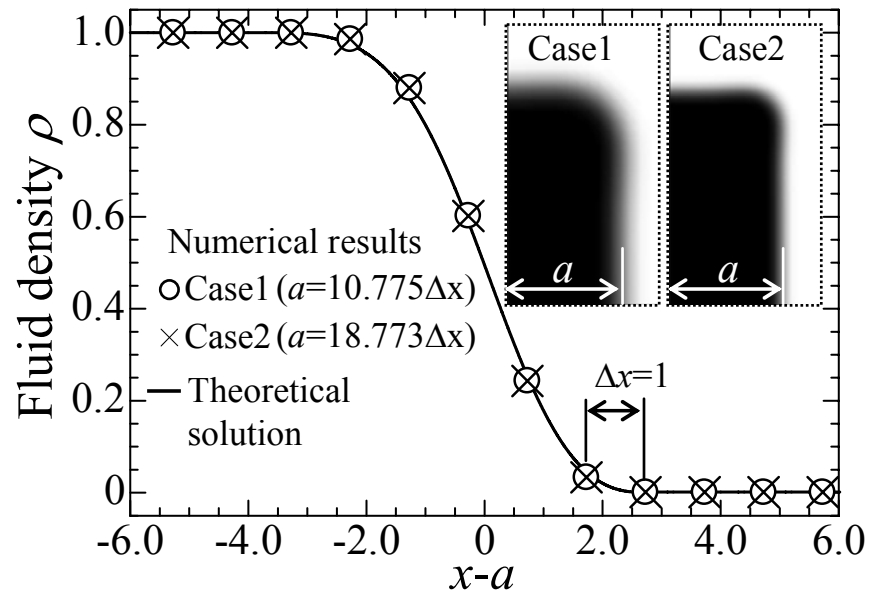

Fig.6 Initial profile of fluid density $\rho$ across liquid-column interface on bottom solid wall.

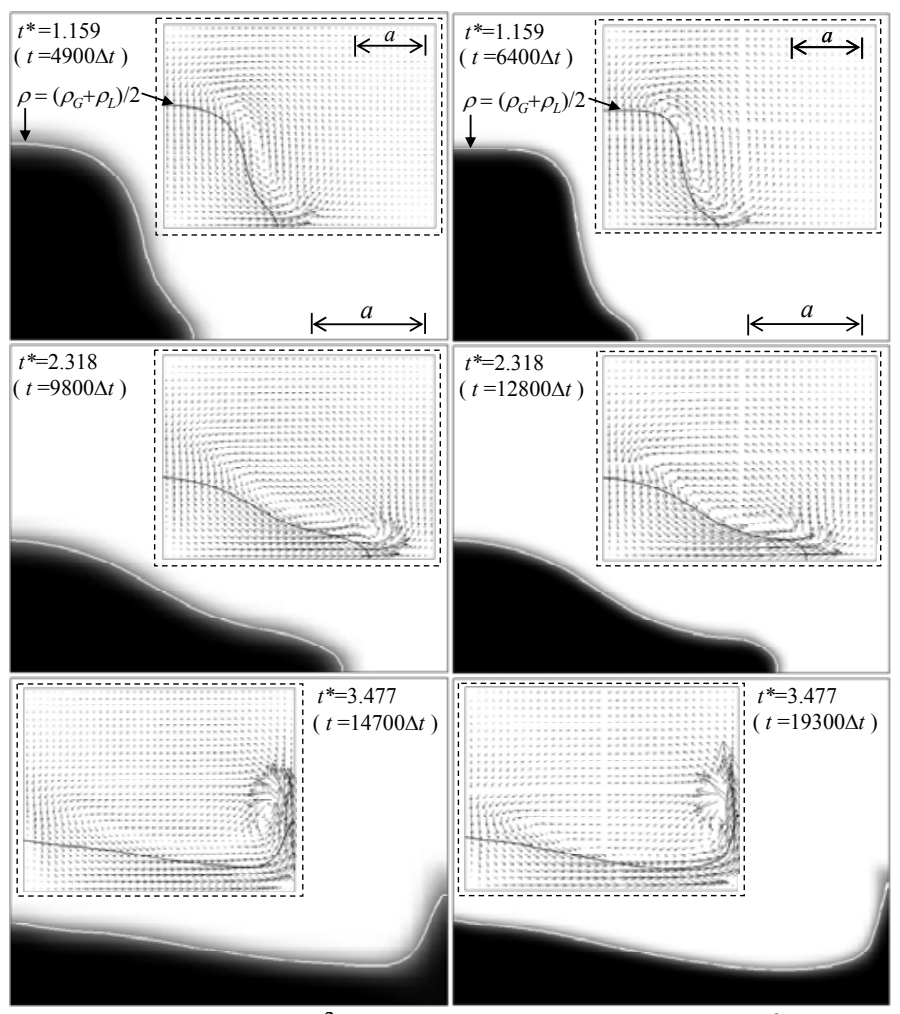

(a) Case1 ( $\left.a=10.775, n^{2}=1.937\right)$ (b) Case2 $\left(a=18.773, n^{2}=1.959\right)$

Fig.7 Snapshots of diffuse-interfacial profile and flow velocity field in collapse of liquid column with initial width $a$ at dimensionless time $t^{*}(\Delta t=0.0125)$. two-dimensional rectangular domain surrounded with non-slip solid walls. In both cases of spatial resolution (Table1), initial column width $a$ was assumed to be equivalent to $146 \mathrm{~mm}$ in airwater system. Fig. 6 shows initial density profiles across diffusive interface on the bottom wall surface. The numerical solutions at $\Delta x=\Delta y=1$ in Eqs.(3)-(5) (symbols) agreed with the theoretical prediction (solid line). As shown in Fig.7, interfacial shapes in both high and low resolutions agreed well with each other at each dimensionless time. Time series of horizontal leading-edge position $X$ and velocity $U$ of column (solid and broken lines in Fig.8 and Fig.9) on a surface of bottom wall also agreed well with other predictions $[14,19,20]$.

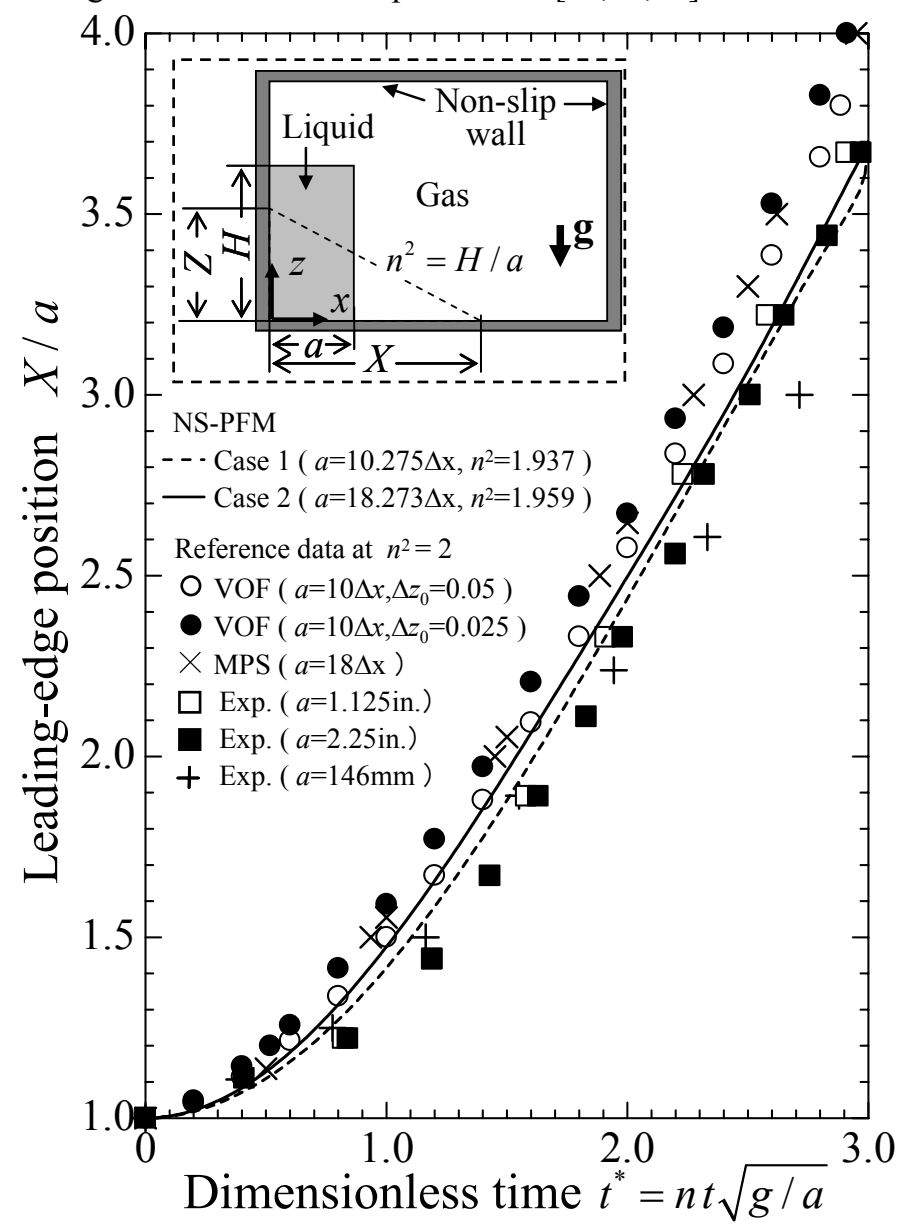

Fig. 8 Time series of horizontal leading-edge position $X$ of 2D collapsing liquid column with width $a$, height $H$, and aspect ratio $n^{2}$ under gravity $\mathbf{g}$ in present and other data.

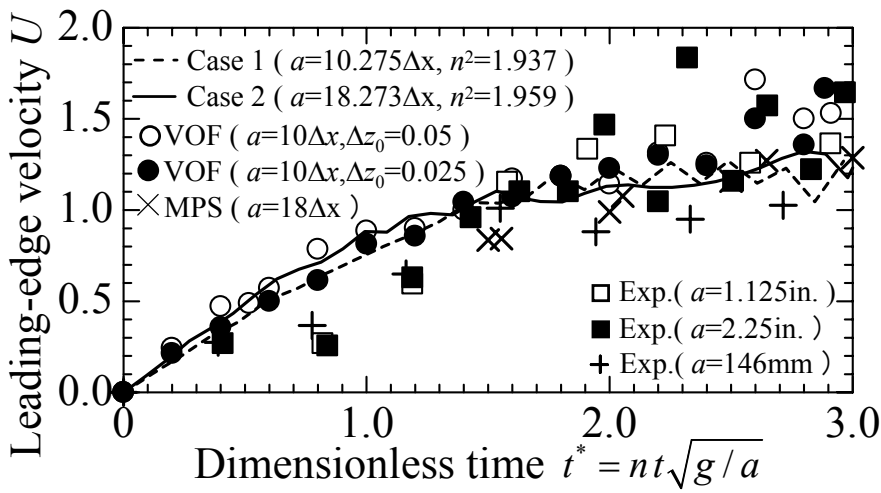

Fig.9 Dimensionless horizontal velocity of leading edge of liquid column with aspect ratio $n^{2}=2$. 


\section{Coalescence of free-fall drop and stagnant liquid film}

As the third example, we simulated three-dimensional coalescence of single drop falling through a stagnant gas into a liquid film sustained on a horizontal solid wall under gravity. The diameter of the drop $d=20 \Delta \mathrm{x}$ in the simulation was equivalent to $10 \mathrm{~mm}$ in actual air-water system. As seen in Fig.10, pressure builds up at a spot between the drop and liquid film at dimensionless time $t^{*}=0.879$ just before contact, while it moved along the surface of bottom plane at $t^{*}=1.001$ after the drop penetrated by half into the liquid film. After fully merged, a ring-shaped vortex was generated by gas jet ejecting from the gap between the drop and film, when an annular single wave propagated outward. In each case of height of drop $H=35,45$, 55 , and 65 , the pressure reached its maximum value before $t^{*}=1$, which was approximately equivalent to initial potential
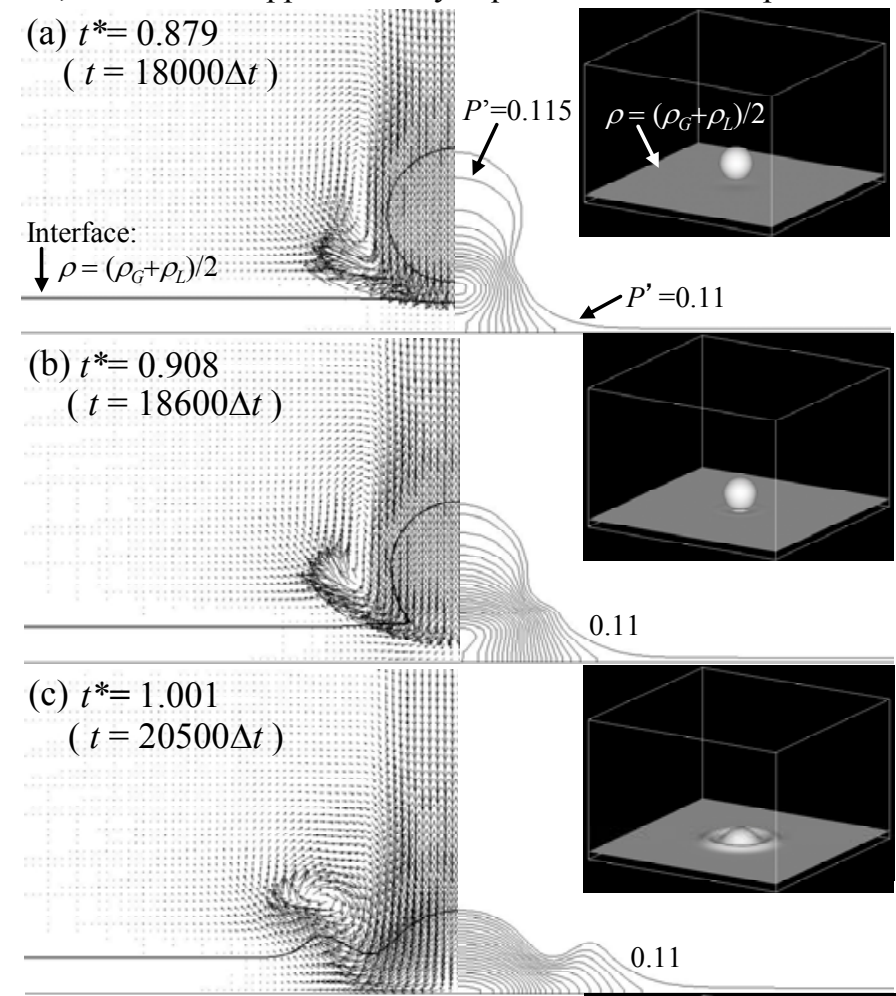

0.11
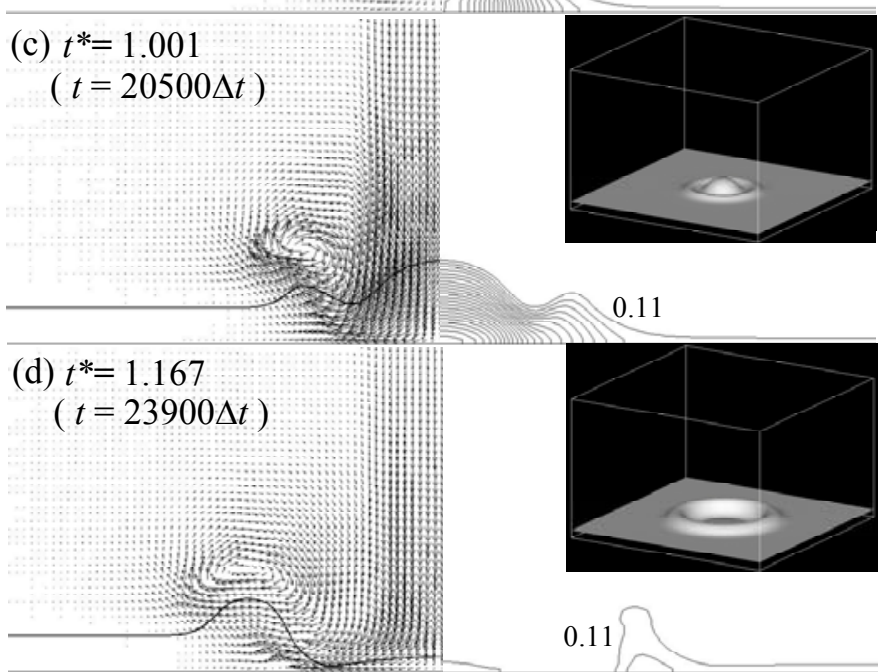

0.11

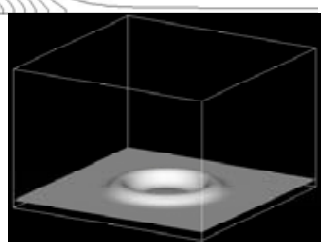

$$
\text { (e) } \begin{aligned}
t^{*} & =1.407 \\
(t & =28800 \Delta t)
\end{aligned}
$$

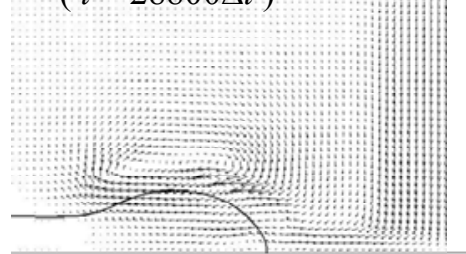

\subsection{1}

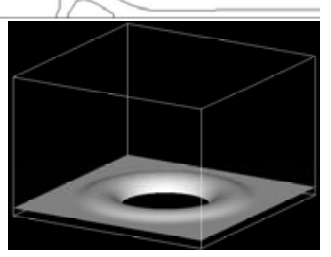

0.11

Fig.10 Snapshots of interfacial profile, cross-sectional flow velocity and pressure fields in coalescence of drop and liquid film at dimensionless time $t^{*}$. energy of the drop (see Fig.11).

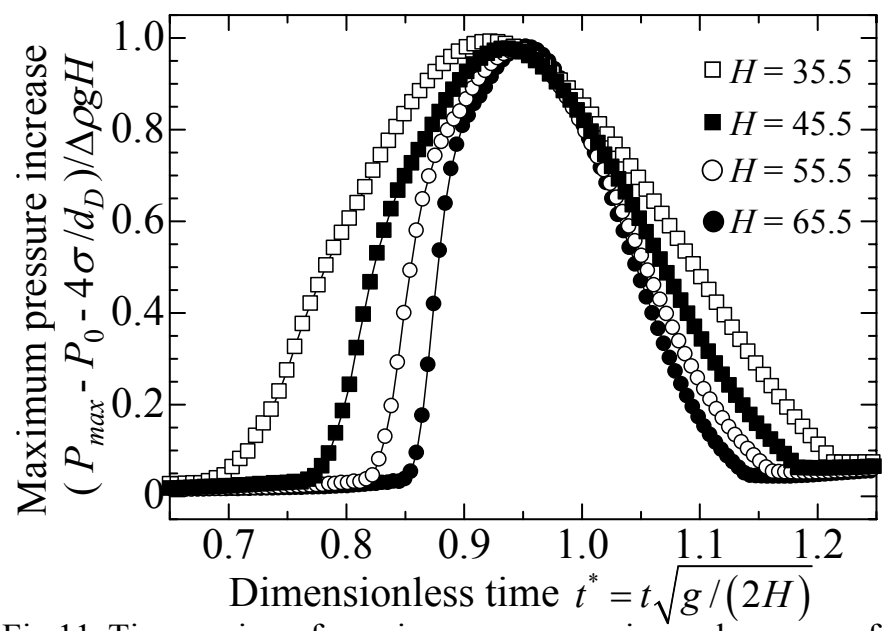

Fig.11 Time series of maximum pressure in coalescence of liquid film and free-fall single drop with diameter $d_{D}$ and initial height position $H$ under gravity $g$ in a stagnant gas.

\section{Bubble nucleation in non-ideal fluid}

This subsection describes a direct numerical simulation of bubble nucleation in a van-der-Waals fluid. The NS-PFM was applied to a case with a condition of $A=B=c=1, \kappa_{S}=0.01$, $k=\mu=0.2, g=0$, and $\Delta t=0.2$ in two dimensions. The stagnant liquid of density $\rho_{L}=0.405$ at temperature $T_{0}=0.293$ in the square area $50^{2} \Delta x \Delta y$ was surrounded with periodic boundaries on both sides, free outflow at the top and non-slip wall on the bottom. A heated section with a constant temperature $T_{H}$ $=T_{0}+1.465 \times 10^{-2}$ and width $L_{H}=10 \Delta x$ was located on the bottom wall set at $T_{\mathrm{W}}=T_{0}$. As shown in Fig.12, a bubble nucleation took place on the heated section. The velocity and temperature gradient in gas phase with $\rho_{G}=0.265$ gradually reduced as the bubble grew into a mushroom-shape.

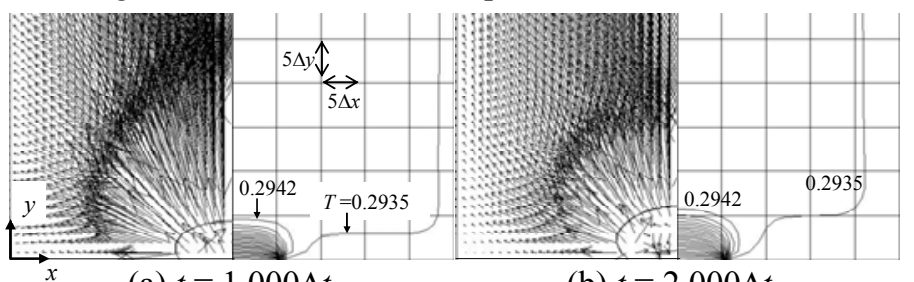

(a) $t=1,000 \Delta t$

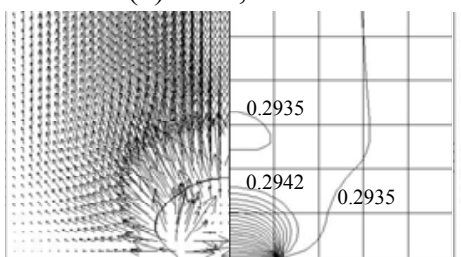

(c) $t=5,000 \Delta t$

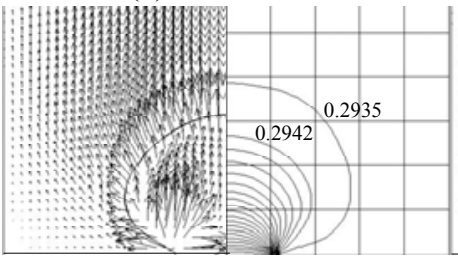

(e) $t=20,000 \Delta t$ (b) $t=2,000 \Delta t$

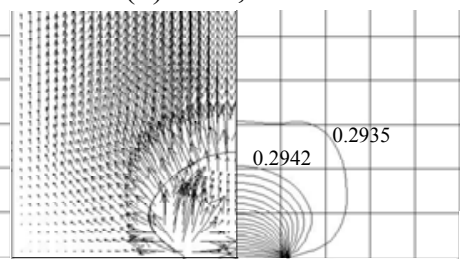

(d) $t=10,000 \Delta t$

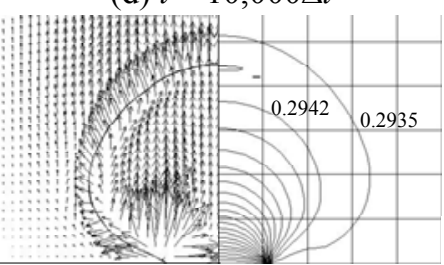

(f) $t=50,000 \Delta t$
Fig.12 Interface profile, velocity and temperature fields around $2 \mathrm{D}$ bubble nucleated on heater with width $L_{H}=10 \Delta x$ in a van-der-Waals fluid at time $t(\Delta x=\Delta y=1, \Delta t=0.2)$. 


\section{CONCLUSIONS}

To simulate the interface motion in two-phase flows, we proposed two versions of phase-field method, NS-PFM, combining Navier-Stokes (NS) equations with phase-field model (PFM) based on the free-energy theory.

The first version of NS-PFM was applied to several flow problems of incompressible, isothermal two-phase fluid with a high density ratio equivalent to that of air-water system. From the numerical results, it was confirmed that (1) the volume flux driven by a local chemical potential gradient in the CahnHilliard equation plays an important role in (a) volume conservation, (b) self-organizing reconstruction of gas-liquid interface, and (c) reduction of numerical diffusion and oscillation, (2) this method gives good predictions of pressure increase inside a bubble caused by the surface tension force, (3) a single liquid drop falling through a stagnant gas and merging into a stagnant liquid film was simulated adequately, and (4) collapse of liquid column under gravity was predicted in good agreement with well-known experimental and numerical data. Another type of NS-PFM was used for a direct numerical simulation of nucleation in non-ideal fluid in the vicinity of critical point. It was demonstrated that the proposed NS-PFM is able to successfully reproduce liquid-vapor interface motion with heat transfer in boiling and condensation.

\section{ACKNOWLEDGMENTS}

A part of this study has been financially supported by the Budget for Nuclear Research of the Ministry of Education, Culture, Sports, Science and Technology, based on the screening and counseling by the Atomic Energy Commission, in 2002-2005.

\section{REFERENCES}

[1] Inamuro, T., Ogata, T., Tajima, S., and Konishi, N., 2004, "A Lattice Boltzmann Method for Incompressible TwoPhase Flows with Large Density Differences," J. Comput. Phys., 198, pp.628-644.

[2] Takada, N., Tomiyama, A., and Hosokawa, S., 2003, "Numerical Simulation of Drops in a Shear Flow by a Lattice-Boltzmann Binary Fluid Model," Comput. Fluid Dyn. J., 12, pp.475-481.

[3] Jacqmin, D., 1999, "Calculation of Two-Phase NavierStokes Flows Using Phase-Field Modeling," J. Comput. Phys., 155, pp.96-127.

[4] Jamet, D., Lebaique, O., Coutris, N. and Delhaye, J.M., 2001, "The Second Gradient Method for the Direct Numerical Simulation of Liquid-Vapor Flows with Phase Change," J. Comput. Phys., 169, pp.624-651.

[5] Seta, T., and Kono, K.., 2004, "Thermal Lattice Boltzmann Method for Liquid-Gas Two-Phase Flows in Two Dimensions," JSME Int. J. Ser.B, 47, pp.572-583.

[6] Bi, Z., and Sekerka, R.F., 1998, 'Phase-Field Model for Solidification of a Binary Alloy,'Physica,A 261, pp.95-106.

[7] Morita,H., Kawakatsu,T., and Doi,M., 2001, "Dynamic Density Functional Study on the Structure of Thin Polymer Blend Films with a Free Surface," Macromolecules, 34, pp.8777-8783.

[8] Cahn, J.W., and Hilliard, J.E., 1958, "Free Energy of a Nonuniform System. I. Interfacial Free Energy," J. Chem. Phys., 28, pp.258- 267.
[9] Brackbill, J.U., Kothe, D.B. and Zemach, C., 1992, "A Continuum Method for Modeling Surface Tension," J. Comput. Phys., 100, pp.335-354.

[10] Kunugi,T., and Satake,S., 1999, "Direct Numerical Simulation of Turbulent Free-Surface Flow," Turbulence and Shear Flow Phenomena-1, pp.621-626, Begell House, New York.

[11] Yabe, T., Xiao, F., and Utsumi, T., 2001, "The Constraind Interpolation Profile Method for Multiphase Analysis," J.Comput. Phys., 169, pp.556-593.

[12] Tryggvason, G., Bunner, B., Esmaeeli, A., Juric, D., AlRawahi, N., Tauber, W., Han, J., Nas, S., and Jan, Y.-J., 2001, "A Front-Tracking Method for the Computations of Multiphase Flow,” J. Comput. Phys., 169, pp.708-759.

[13] Chang, Y.C., Hou, T.Y., Merriman, B., and Osher, S., 1996, "A Level Set Formulation of Eulerian Interface Capturing Methods for Incompressible Fluid Flows," J. Comput. Phys., 124, pp.449-464.

[14] Hirt, C.W. and Nichols, B.D., 1981, "Volume of Fluid (VOF) Method for the Dynamics of Free Boundaries," J. Comput. Phys., 39, pp.201-225.

[15] Swift, M. R., Orlandini, E., Osborn W. R., and Yeomans, J. M., 1996, "Lattice Bolztammn Simulations of Liquid-Gas and Binary Fluid Systems," Phys.Rev., E 54, pp.5041-5052.

[16] Chen, S. and Doolen, G.D., 1998, "Lattice Boltzmann Method for Fluid Flows," Annu. Rev. Fluid Mech., 30, pp. 329-364.

[17] Succi,S., 2001, The Lattice Boltzmann Equation for Fluid Dynamics and Beyond, Oxford University Press, New York.

[18] Kawamura, T., and Kuwahara, K., 1984, "Computation of High Reynolds Number Flow around a Circular Cylinder with Surface Roughness," AIAA Paper, 84-0340.

[19] Martin, J.C., and Moyce, W.J., 1952, “An Experimental Study of the Collapse of Liquid Columns on a Rigid Horizontal Plane," Philos. Trans. Roy. Soc. London, Ser.A, 244, pp.312-324.

[20] Koshizuka, S., and Oka, Y., 1996, "Moving-Particle SemiImplicit Method for Fragmentation of Incompressible Fluid," Nucl. Sci. Eng., 123, pp.421-434. 